\section{Un nouvel acteur dans la neutralisation intracellulaire des adénovirus par les anticorps}

Karim Benihoud
Vectorologie et thérapeutiques anticancéreuses, UMR 8203, CNRS, Université Paris-Sud, Gustave Roussy,

Université Paris-Saclay, 114 rue Édouard Vaillant, 94805 Villejuif, France.

karim.benihoud@gustaveroussy.fr
> Les adénovirus ( $\mathrm{Ad}$ ) sont des virus à ADN, à capside non enveloppée, responsables chez l'homme d'infections souvent asymptomatiques. Dans le cas de l'Ad de sérotype 5 (Ad5), l'infection des cellules hôtes débute par l'attachement d'une protéine de capside, la fibre, sur le coxsackie and adenovirus receptor (CAR), suivi d'une seconde interaction entre une autre protéine de capside, la base du penton, et les intégrines, qui conduit à l'internalisation de la particule virale. Très rapidement, le virus perd ses fibres, expose une protéine interne de capside, la protéine VI (pVI), capable de lyser la membrane des endosomes; la particule virale en partie désassemblée va alors migrer via le réseau de microtubules jusqu'au noyau. L'Ad5 peut être modifié par génie génétique en un vecteur capable de transférer des gènes à visée anti-tumorale ou vaccinale. Cependant, l'efficacité de ces vecteurs est limitée par la forte prévalence des anticorps ( $A c)$ anti-Ad chez I'homme. Ceux-ci sont dirigés contre les protéines majeures de capside: fibre, base du penton et hexon, les Ac anti-hexon étant majoritaires [1]. Les Ac anti-Ad peuvent neutraliser les particules virales dans le milieu extracellulaire, mais agissent également au niveau intracellulaire [2]. L'équipe de Léo $C$. James a révélé que les complexes $A c-A d$ activent, via la région constante (Fc) des Ac (Figure IA), le récepteur cytosolique TRIM21 (tripartite motif-containing protein 2l), conduisant à la dégradation de l'Ad par le protéasome [3].
Neutralisation des adénovirus par les anticorps associés aux composés $\mathrm{Cl}$ et C4 du complément

Récemment, l'équipe de Léo C. James a cherché à mieux comprendre les mécanismes à l'origine de la neutralisation intracellulaire des Ad par les Ac en analysant la contribution du complément [4], un ensemble de protéines sériques qui s'activent en cascade selon différentes voies. En particulier, l'activation de la voie classique est déclenchée par les complexes immuns qui recrutent le complexe $\mathrm{Cl}$ (constitué par les sousunités $\mathrm{Clq}, \mathrm{Clr}$ et $\mathrm{Cls}$ ), aboutissant à l'activation de la protéine $\mathrm{C4}$. Celle-ci, après interaction avec le composant $\mathrm{C2}$, conduit à la production d'une C3 convertase ( $C 4 b C 2 a)$, puis à l'activation de différents composés ( $55-C 9)$, déclenchant les fonctions effectrices du complément (Figure 1B).

Dans un premier temps, l'équipe de chercheurs a analysé comment un Ac monoclonal dirigé contre l'hexon (composant principal de la capside) neutralisait I'Ad5 en présence de sérum humain'. $\varepsilon n$ accord avec leurs résultats précédents [3], les chercheurs ont montré que l'Ad5 était bien neutralisé par les Ac dans les cellules exprimant TRIM21, mais qu'il l'était également dans des cellules déficientes en TRIM21, ce qui suggérait l'existence d'un autre mécanisme de neutralisation. Ils ont donc évalué le rôle de la voie classique du complément en s'intéressant à l'étape initiale de cette voie, l'interaction des Ac avec $\mathrm{Clq}$. Les Ac anti-hexon mis en présence

\footnotetext{
${ }^{1}$ Pour rappel, le sérum contient les composants du complément.
}

d'un sérum humain déficient en Clq sont apparus incapables de neutraliser l'Ad5. De même, un Ac anti-hexon incapable d'interagir avec Clq s'est avéré également incapable de neutraliser l'Ad5. Ayant démontré le rôle de $\mathrm{Clq}$ dans la neutralisation des Ad, les chercheurs ont analysé si d'autres acteurs moléculaires de la voie classique étaient impliqués dans la neutralisation de l'Ad par les Ac. Des expériences de déplétion de sérum humain en différents facteurs du complément ont non seulement confirmé que Clq mais aussi révélé que $C 4$ étaient tous deux indispensables à la neutralisation des Ad par les Ac, contrairement au composé C2, situé en aval du C4 dans la cascade de la voie classique (Figure 1B). Ces résultats démontrent l'existence d'un nouveau mécanisme de neutralisation par les Ac, indépendant de TRIM2l, et impliquant $\mathrm{Clq}$ et $\mathrm{C} 4$ indépendamment de leur rôle dans la formation de la C3 convertase.

\section{Déshabillage interdit pour}

l'adénovirus, un rôle inattendu du C4 ! Ayant observé que l'incubation de l'Ad avec I'Ac anti-hexon en présence de sérum humain conduisait à un clivage de C4 en C4a et C4b, les chercheurs ont évalué si la neutralisation des Ad par le complément découlait d'un effet de C4a sur les cellules cibles ou résultait de la fixation de C4b sur l'Ad. L'analyse de l'expression de transgènes après co-incubation des cellules avec un Ad codant un transgène et un $\mathrm{Ad}$ recouvert de $C 4 b$ codant un autre transgène a montré que seul l'Ad recouvert de C $4 b$ était neutralisé. Ceci permettait 
A

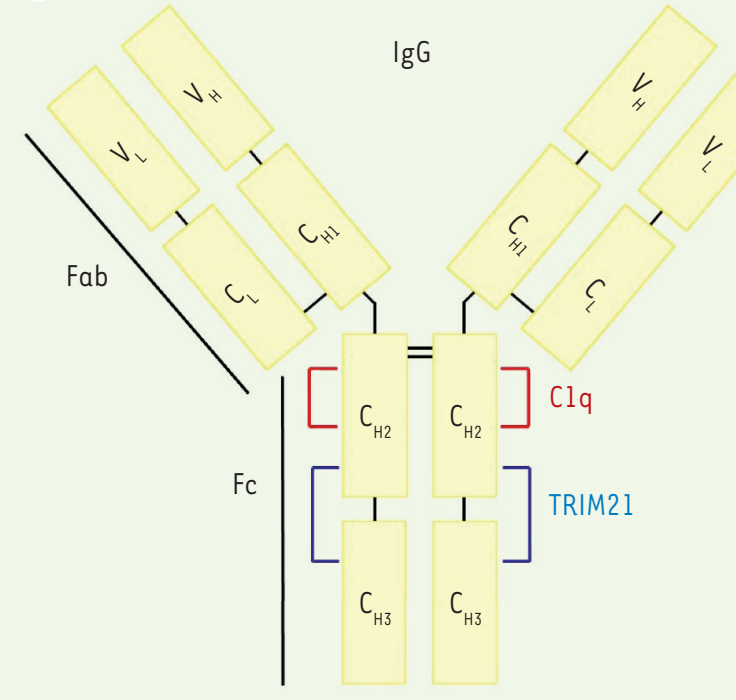

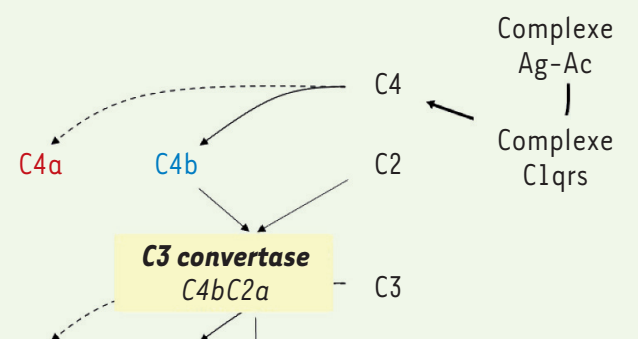

Figure 1. Domaines d'interaction des anticorps et voie classique d'activation du complément. A. Le schéma indique les domaines variables (V) et constants $(C)$ des chaînes lourdes $(H)$ et légères $(L)$ d'un anticorps de type immunoglobuline $(\mathrm{Ig} G)$. La région Fc contient les zones d'interaction avec les protéines Clq et TRIM21. B. L'interaction des complexes Ag-Ac avec le complexe Clqrs déclenche l'activation en cascade de différents composants du complément et la production de différentes molécules impliquées dans l'inflammation (en rouge), jouant un rôle d'opsonines (en bleu) ou impliquées dans la lyse des microorganismes pathogènes (en vert).

d'éliminer l'hypothèse d'une modification des cellules cibles et suggérait un rôle de $\mathrm{C} 4 \mathrm{~b}$ dans le processus de neutralisation. Après incubation des Ad avec des $A c$ en présence de sérum humain (ou de Clq et C4 purifiés), les chercheurs ont observé que le C4 était associé aux particules virales, ce qui suggérait que le fragment $C 4 b$ se fixait aux particules adénovirales et que cette fixation permettait leur neutralisation. Les étapes de fixation aux cellules cibles et d'internalisation des particules virales ne sont pas affectées par la fixation de C4b, mais les particules virales opsonisées par C4b deviennent incapables de libérer les fibres, une étape du désassemblage de la capside qui a lieu au cours de l'infection virale. L'étude du trafic intracellulaire par microscopie confocale montre que les particules virales opsonisées par $\mathrm{C} 4 \mathrm{~b}$ et présentes dans les endosomes n'exposent pas la protéine adénovirale pVI à la surface de leur capside. II en résulte un défaut de lyse de la membrane de l'endosome, qui bloque la libération des particules virales dans le cytosol.

\section{Action synergique du composant C4} et du récepteur TRIM21 dans la neutralisation intracellulaire des adénovirus par les anticorps Après avoir montré le rôle de C4 dans la neutralisation des Ad à l'aide de modèles cellulaires, l'équipe de Léo C. James a analysé l'importance physiologique de ce mécanisme en administrant, par voie intraveineuse, des vecteurs adénoviraux à des souris ayant des Ac anti-hexon. Les chercheurs ont ainsi montré que le transfert de gènes par un vecteur adénoviral est plus efficace chez les souris génétiquement déficientes en C4 que chez les souris témoins, ce qui confirme le rôle de C4 dans la neutralisation intracellulaire des $\mathrm{Ad}$ in vivo. Le rôle de $\mathrm{Clq}$ dans ce phénomène est également étayé, puisque le transfert de gènes chez les souris témoins est augmenté si la région $\mathrm{Fc}$ de l'Ac anti-hexon est mutée pour empêcher son interaction avec le Clq. En se fondant sur leurs travaux précédents montrant la neutralisation intracellulaire des $\mathrm{Ad}$ recouverts d'Ac suite à l'interaction de ces derniers avec le récepteur TRIM2l via leur région $\mathrm{Fc}[5]$, les chercheurs ont montré que l'administration de l'Ac anti-hexon muté pour empêcher son interaction avec le $\mathrm{Clq}$ à des souris déficientes en TRIM2l aboutissait à un transfert de gènes comparable à celui observé chez des souris dépourvues d'Ac anti-hexon. Ces résultats indiquent que TRIM2l et $\mathrm{Clq} / \mathrm{C} 4$ mettent en jeu des mécanismes indépendants de neutralisation intracellulaire par les Ac, ce qui est en accord avec le fait que $\mathrm{Clq}$ et TRIM2l interagissent avec différentes régions de la région $\mathrm{Fc}$ des $\mathrm{Ac}$ (Figure $1 \mathrm{~A}$ ).

\section{Vers une inhibition des fonctions neutralisantes du complément} et de TRIM21

Ainsi, alors que l'infection des cellules par l'Ad en absence d'Ac conduit à un échappement de l'endosome et au transfert du génome viral dans le noyau (Figure 2A), l'infection en présence d'Ac 
A

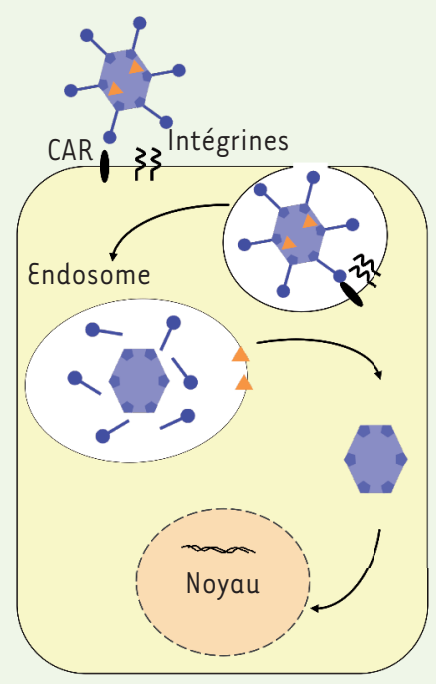

B

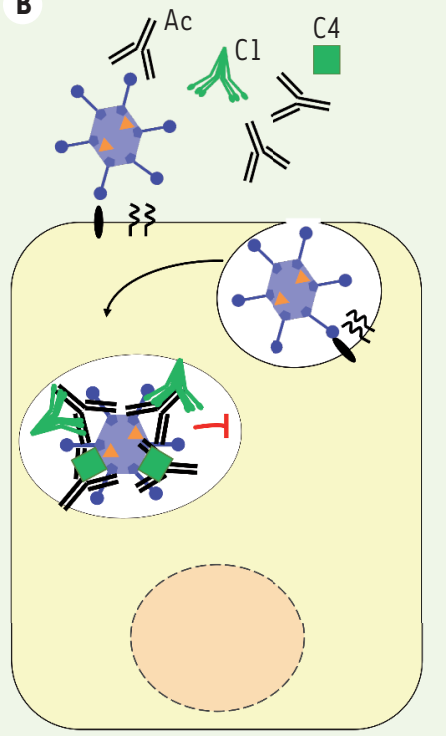

C

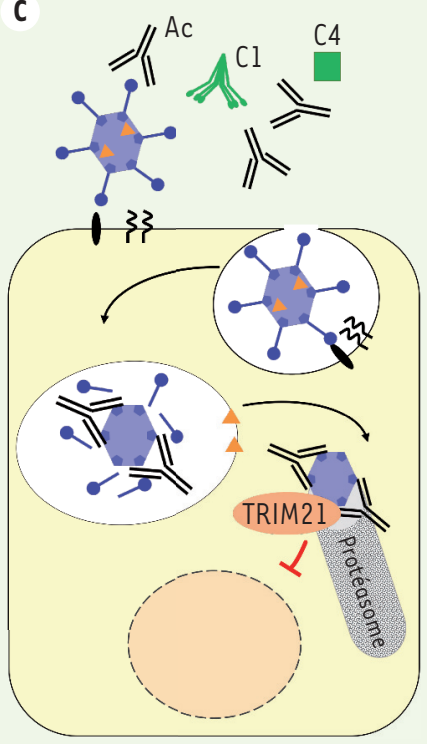

Figure 2. Mécanismes de neutralisation intracellulaire des adénovirus par les anticorps. A. Étapes de l'infection des cellules par les Ad, en l'absence d'Ac. B.C. Rôle de C4 (B) ou de TRIM21 (C) lors de l'infection des cellules par les Ad en présence d'Ac. CAR : coxsackie and adenovirus receptor.

conduit à une neutralisation intracellulaire des Ad par le composé C4, capable d'inhiber le désassemblage de la capside virale et l'échappement de l'endosome (Figure 2B) par un mécanisme très proche de celui décrit précédemment pour certaines défensines [6]. Cette neutralisation intracellulaire des Ad par les Ac a lieu à une étape plus précoce que celle enclenchée par TRIM21, reposant sur la détection et la dégradation des complexes Ad-Ac dans le cytosol (Figure 2C). Les travaux de Bottermann et al. ont révélé un seuil différent de mise en œuvre de ces mécanismes, le virus étant neutralisé par le complément lorsque de fortes concentrations d'Ac anti-Ad sont présentes, et par le récepteur TRIM2l pour de faibles concentrations d'Ac. Le fait que l'Ad préincubé avec un $A c$ anti-hexon dépourvu de sa région $\mathrm{Fc}$ (i.e., comportant uniquement la région Fab) ou muté dans sa région
Fc afin d'empêcher son interaction avec Clq et TRIM2l échappe à la neutralisation intracellulaire par les Ac ouvre de nouvelles perspectives pour le transfert de gènes par Ad. Ainsi, des Ac de type single chain fragment variable (scFv) ou des protéines à domaines ankyrine [7] pourraient être utilisés pour masquer les sites antigéniques de la capside virale, prévenant ainsi la fixation d'Ac anti-Ad et la neutralisation intracellulaire dépendant de C4 et TRIM2l. Des modifications ciblées de la capside capables de bloquer l'interaction avec les Ac pourraient également être envisagées [8]. $\diamond$

A new actor involved in antibodydependent intracellular neutralization of adenovirus

\section{LIENS D'INTÉRÊT}

L'auteur déclare n'avoir aucun lien d'intérêt concernant les données publiées dans cet article.

\section{RÉFÉRENCES}

1. Sumida SM, Truitt DM, Lemckert AA, et al. Neutralizing antibodies to adenovirus serotype 5 vaccine vectors are directed primarily against the adenovirus hexon protein. J Immunol 2005 ; 174 : 7179-85.

2. Wohlfart C. Neutralization of adenoviruses: kinetics, stoichiometry, and mechanisms. J Virol 1988 ; 62 : 2321-28.

3. Mallery DL, McEwan WA, Bidgood SR, et al. Antibodies mediate intracellular immunity through tripartite motif-containing 21 (TRIM21). Proc Natl Acad Sci USA 2010 ; $107: 19985-90$.

4. Bottermann M, Foss S, Caddy SL, et al. Complement C4 prevents viral infection through capsid inactivation. Cell Host Microbe 2019 ; 25 : 617-29.

5. Bottermann M, Foss S, Tienen LM van, et al. TRIM21 mediates antibody inhibition of adenovirus-based gene delivery and vaccination. Proc Natl Acad Sci USA 2018 ; $115: 10440-45$.

6. Smith JG, Silvestry M, Lindert S, et al. Insight into the mechanisms of adenovirus capsid disassembly from studies of defensin neutralization. PLoS Pathog 2010 ; 6: el000959.

7. Schmid M, Ernst P, Honegger A, et al. Adenoviral vector with shield and adapter increases tumor specificity and escapes liver and immune control. Nat Commun 2018 ; 9 : 450.

8. Rojas LA, Condezo GN, Moreno R, et al. Albuminbinding adenoviruses circumvent pre-existing neutralizing antibodies upon systemic delivery. J Control Release 2016 ; 237 : 78-88.

\section{Retrouvez toutes les Actualités de la Myologie sur les sites de :}

la Société Française de Myologie

www.sfmyologie.org
Sfm

www.filnemus.fr

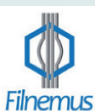

